\title{
Bilateral Symmetrical Mucocele of the Lower Lip: Report of a Rare Clinical Presentation
}

\author{
${ }^{1}$ Hamed Mortazavi, ${ }^{2}$ Hamid Reza Khalighi, ${ }^{3}$ Maryam Baharvand, ${ }^{4}$ Majid Eshghpour
}

\section{ABSTRACT}

Aim: Oral mucocele $(\mathrm{OM})$ is a common benign lesion in the oral cavity. We aimed to present an unusual clinical feature of OM.

Materials and methods: We report an 8-year-old girl presented with symmetrical bilateral masses on her lower lip with a known history of lip biting during school hours. After surgical removal of the lesions, histopathologic evaluation showed dilatation of minor salivary gland ducts because of mucus retention, and diagnosis of mucocele was established.

Results: According to our data, presence of bilateral mucocele was reported once before, which was an asymmetrical occurrence of lower lip mucocele causing malocclusion in a 3-year-old child.

Conclusion: Although rare, oral mucocele may present as bilateral symmetrical lesions.

Keywords: Mucocele, Bilateral, Symmetrical.

How to cite this article: Mortazavi $\mathrm{H}$, Khalighi HR, Baharvand M, Eshghpour M. Bilateral Symmetrical Mucocele of the Lower Lip: Report of a Rare Clinical Presentation. Int J Experiment Dent Sci 2014;3(2):92-94.

Source of support: Nil

Conflict of interest: None

\section{INTRODUCTION}

Oral mucocele $(\mathrm{OM})$ is a common lesion defined as a swelling caused by accumulation of saliva at the site of a traumatized or obstruction of minor salivary gland duct due to sialolith, dense mucosa or scar tissue formation around an injured duct. The incidence of this entity is pretty high, 2.5 lesions per 1000 patients with no significant sex predilection. It is frequently seen in the second decade of life

\footnotetext{
${ }^{1,3}$ Associate Professor, ${ }^{2,4}$ Assistant Professor

${ }^{1-3}$ Department of Oral and Maxillofacial Medicine, Shahid Beheshti University of Medical Sciences Dental School Tehran, Iran

${ }^{4}$ Department of Oral and Maxillofacial Surgery, Mashhad University of Medical Sciences Dental School, Mashhad, Iran
}

Corresponding Author: Maryam Baharvand, Associate Professor, Department of Oral and Maxillofacial Medicine Shahid Beheshti University of Medical Sciences Dental School Daneshjoo Blvd, Tabnak St, Chamran Highway, Tehran, Iran Zip Code: 1983963113, Phone: +98-2129902311, e-mail: marbahar@gmail.com and is rare among children. Mucoceles are divided into two subgroups: extravasation and retention types. ${ }^{1-3}$ According to Bagen et al, extravasation type (95\%) is more common than retention type $(5 \%) .{ }^{4}$ Mucocele is usually characterized by an asymptomatic, bluish, soft and transparent cystic swelling which frequently resolves spontaneously. However, Luiz reported symptomatic lesions. ${ }^{5}$ In most cases, extravasation mucoceles are seen on the lower lip whereas; retention mucoceles are found at any other location of the mouth. ${ }^{2}$ Mucoceles are usually single and rarely larger than $1.5 \mathrm{~cm}$ in diameter, however multiple lesions may be present at a time. ${ }^{6}$ The aim of this report was to present the first case of bilateral symmetrical mucocele of the lower lip.

\section{CASE REPORT}

An 8-year-old girl was referred to the Department of Oral and Maxillofacial Medicine with the chief complaint of two separate and simultaneous swellings on her lower lip (Fig. 1). The patient was aware of the swellings from 6 months ago with episodic fluctuations in their size. Intraoral examination revealed two pink-colored, sessile, soft to firm, asymptomatic nodules measuring $5 \times 5 \mathrm{~mm}$ on the labial mucosa. The lesions were similar in size, shape, consistency and color. The left lesion exuded little viscous saliva-like liquid, when punctured by an 18-guage needle. The patient had a positive history of lip biting especially during school time. No history of systemic diseases was obtained. According to the provisional diagnosis of mucocele, the patient underwent excisional biopsy of the masses under local anesthesia. Pathologic examination revealed a pseudo cyst surrounding mucous material. Infiltration of chronic inflammatory cells and dilated salivary ducts were also prominent (Fig. 2). After 16 months, there was no evidence of recurrence.

\section{DISCUSSION}

Jahanshahi et al and Tal et al ${ }^{6,7}$ have reported previous cases of multiple labial mucocele, however, presence of lower lip mucocele as bilateral symmetrical lesions is extremely rare. According to our data, bilateral but not symmetrical lower lip mucoceles were first reported by Meechan et al in $1987 .{ }^{8}$

In contrast to our case, Jahanshahi et al showed that multiple mucoceles were more common on the upper lip than the lower lip with upper lip to lower lip ratio of $5: 1{ }^{6}$ 

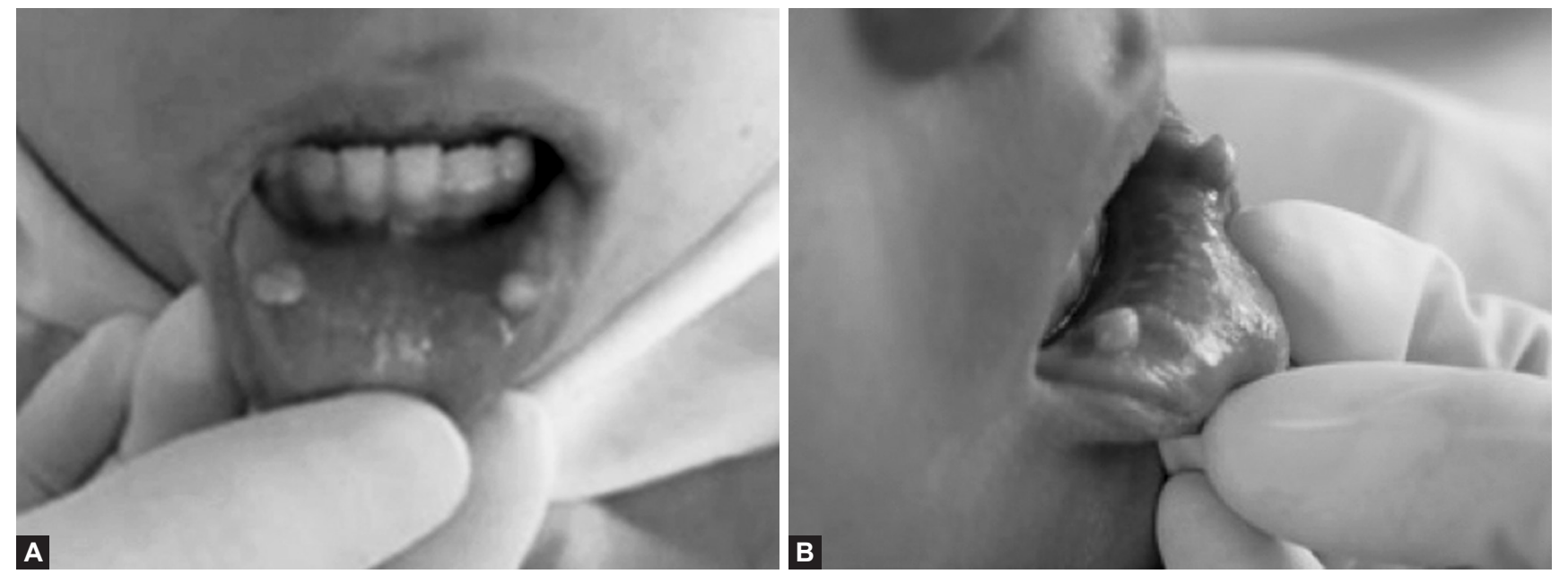

Figs 1A and B: Symmetrical nodules on the lower lip

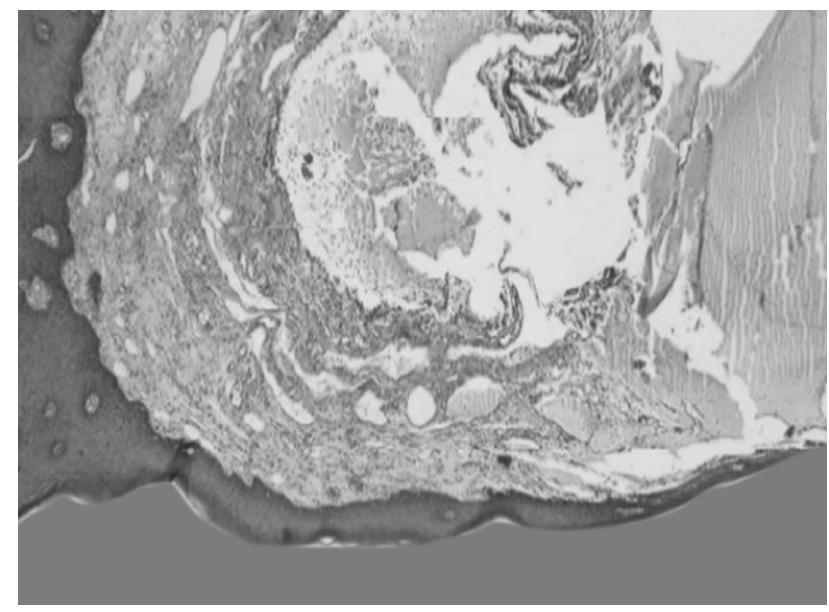

Fig. 2: Histopathological examination shows pseudo cyst surrounding the mucous material and inflammatory cells. (Hematoxylin and Eosin, 40x magnification)

Our case is unique in terms of being double and symmetrical and similarity in lesions' size and shape.

Two crucial factors are involved in the etiology of mucoceles: trauma and obstruction of salivary gland ducts. ${ }^{2}$ In our patient, the history of trauma during school hours was evident.

Tal et al described two possible explanations for the presence of multiple mucoceles, either the duct blockage by viscous saliva, or weakness in the structure of ducts. ${ }^{7}$

Diagnosis of mucocele is mainly based on its characteristic clinical appearance with consideration of some crucial points: location of the lesion, history of trauma, bluish color and lesion's consistency.

The differential diagnosis of mucocele includes: fibroma, lipoma, phlebolith, abscess, hemangioma and salivary gland tumors. ${ }^{2,3,5}$

Fibroma is one of the most common lesions in the oral cavity. Although, the irritation fibroma can occur in any site of oral cavity, the most common location is the buccal mucosa. The lesion typically appears as an asymptomatic, firm, smooth-surfaced pink nodule. They vary in size; however, most fibromas are $1.5 \mathrm{~cm}$ or less in diameter.
In contrast to mucocele, fibroma does not show episodic fluctuations in size over time.

Oral lipoma is usually a soft, nodular mass that can be sessile or pedunculated. Most patients are 40 years of age or older; presence of this lesion is very rare in children.

Hemangioma is the most common tumor of infancy, occurring in about $10 \%$ of 1-year-old children; most commonly observed in the tongue, while mucocele involve lips more frequently. Hemangioma feels less firm to palpation, whereas mucoceles are elastic and fluctuant masses unless they are draining or become chronic with a surrounding fibrous tissue. When located superficially, hemangioma produces a deep blue (if cavernous in origin) to a purple (when originates from capillaries) coloration, while a superficial mucocele appears with a bluish hue if any. Cavernous hemangiomas blanch under pressure due to their emptiability nature (positive diascopy test), but this is not the case for mucocele. Minor salivary gland tumors are most commonly found in the palate and because of their neoplastic nature and progressive growth they do not have a history of episodic fluctuations in size. ${ }^{1,2}$

Our lesions were soft to firm asymptomatic nodules on the lower lip mucosa with a mucous like discharge when aspirated. All of the above characteristics were compatible with clinical diagnosis of mucocele.

Conventional therapy is surgical extirpation of the lesion together with the adjacent acini down to the underneath muscle. Micro marsupialization with a silk suture through the largest diameter of the lesion held in place for 5 to 7 days is a simple and safe method to avoid extra damage to the surrounding structures. Because of their high water content, mucoceles are effectively removed by $\mathrm{CO}_{2}$ laser with minimal postoperative complications. Some authors mention cryosurgery with encouraging results to treat oral mucocele. Others inject highly potent corticosteroids into the lesions to make them regress. ${ }^{3,5,9}$ Some mucoceles regress 
without any treatment intervention. The key point to avoid recurrence following surgery is to remove the adjacent acini and the lesion down to the muscular layer. Special care should be made not to harm the surrounding glandular ducts while suturing to avoid appearance of satellite lesions. ${ }^{1,2}$ Our patient did not show any signs of recurrence after 16 months of follow-up.

\section{CONCLUSION}

From the analysis of the data collected during clinical examination, the dentist can construct a list of differential diagnoses, listing the most probable disease that could cause that particular lesion. This procedure should lead to the final diagnosis of the disease and indicate the most appropriate treatment. ${ }^{10}$

Oral mucocele may have unusual presentation, such as bilateral and symmetrical lesions.

\section{REFERENCES}

1. Chi AC, Lambert PR 3rd, Richardson MS, Neville BW. Oral mucoceles: a clinicopathologic review of 1,824 cases, including unusual variants. J Oral Maxillofac Surg 2011;69(4):1086-1093.
2. Khandelwal S, Patil S. Oral mucoceles: review of the literature. Minerva Stomatol 2012;61(3):91-99.

3. Ata-Ali J, Carrillo C, Bonet C, Balaguer J, Peñarrocha M, Peñarrocha M. Oral mucocele: review of the literature. J Clin Exp Dent 2012;2(1):e18-e21.

4. Bagán Sebastián JV, Silvestre Donat FJ, Peñarrocha Diago M, Milián Masanet MA. Clinicopathological study of oral mucoceles. Av Odontoestomatol 1990;6(7):389-395.

5. Luiz AC, Hiraki KR, Lemos CA Jr, Hirota SK, Migliari DA. Treatment of painful and recurrent oral mucoceles with a highpotency topical corticosteroid: a case report. J Oral Maxillofac Surg 2008;66(8):1737-1739.

6. Jahanshahi G, Shirani AM, Khozeimeh F. Multiple mucous retention cyst (Mucocele) of the oral mucosa: a case report. Dent Res J 2007;4(2):111-113.

7. Tal H, Altini M, Lemmer J. Multiple mucous retention cysts of the oral mucosa. Oral Surg Oral Med Oral Pathol 1984;58(6): 692-695.

8. Meechan JG, Blair GS. Bilateral lower lip mucoceles: cause of functional malocclusion in a 3-year-old child. ASDC J Dent Child 1986;53(5):386-387.

9. Ohta N, Fukase S, Suzuki Y, Aoyagi M. Treatment of salivary mucocele of the lower lip by OK-432. Auris Nasus Larynx 2011; 38(2):240-243.

10. Saini R. Oral lesions: A true clinical indicator in human immunodeficiency virus. J Nat Sc Biol Med 2011;2:145-150. 